\title{
Corrigendum: Glycobiology of neuroblastoma: impact on tumor behavior, prognosis, and therapeutic strategies
}

\section{Nora Berois ${ }^{1}$ and Eduardo Osinaga ${ }^{1,2 *}$}

1 Laboratorio de Glicobiología e Inmunología Tumoral, Institut Pasteur de Montevideo, Montevideo, Uruguay

2 Departamento de Inmunobiología, Facultad de Medicina, Universidad de la República, Montevideo, Uruguay

${ }^{*}$ Correspondence: eosinaga@fmed.edu.uy

Edited and reviewed by:

Adriane Regina Todeschini, Universidade Federal do Rio de Janeiro, Brazil

Keywords: neuroblastoma, glycosylation, gangliosides, polysialic acid, galectin-1, glycosyltransferases, immunotherapy

\section{A corrigendum on}

Glycobiology of neuroblastoma: impact on tumor behavior, prognosis, and therapeutic strategies

by Berois $N$ and Osinaga E. Front Oncol (2014) 4:114. doi: 10.3389/fonc.2014.00114

The glycosyltransferases B4GALNT3 and B4GALT3 were mistakenly analyzed as B4GALNT3 in this review. The Table 1 was changed and the text in the last paragraph of "Glycosyltransferases as tumor markers in NB patients" was modified as follows. $\beta 1,4-N$-acetylgalactosaminyltransferase III (B4GALNT3) cloned by Sato et al., has been described as expressed in stomach, colon, and testis (136). This enzyme can transfer GalNAc residues to nonreducing terminal GlcNAc- $\beta$ leading to the synthesis of GalNAc $\beta 1-4 \mathrm{GlcNAc}$ (also known as LacdiNAc or LDN), which is a unique terminal structure in the outer chain moieties of human $N$-glycans (137), and also in $O$-linked oligosaccharide structures (138). The largest amount of B4GALNT3 transcripts were found in gastric tissues, followed by colon, testis, and adrenal glands (136). Gastric expression of B4GALNT3 was found regulated by cellular differentiation (139). In the human colon, Huang et al. reported that B4GALNT3 is up-regulated in primary tumors comparing with the normal mucosa (140). They performed in vitro and in vivo experiments showing that overexpression of this enzyme increases malignant phenotype of colon cancer cells, and these phenotypic changes are associated with enhanced integrin and mitogen-activated protein kinase (MAPK) signaling, suggesting that B4GALNT3

Table 1 | Glycosyltransferases as neuroblastoma (NB) tumor markers

\begin{tabular}{|c|c|c|c|}
\hline Enzyme & Method/sample & Clinical significance & Reference \\
\hline$\beta 1,4-N$-acetylgalactosaminyltransferase & ICC/bone marrow & Molecular marker of metastatic NB & J Clin Oncol; 4:363-369 (1986) \\
\hline \multirow[t]{3}{*}{ (GD2 synthase) } & RT-PCR ECL/bone marrow & Molecular marker of metastatic NB & Cancer; 92:924-931 (2001) \\
\hline & qRT-PCR/bone marrow & Marker for minimal residual disease & J Clin Oncol; 21:1087-1093 (2003) \\
\hline & qRT-PCR/bone marrow-PB & Prognostic marker (poor outcome) & Int J Cancer; 123:2849-2855 (2008) \\
\hline$N$-acetylglucosaminyltransferase $V(G n T-V)$ & qRT-PCR/primary tumor & Prognostic marker (better outcome) & FEBS Lett; 580:627-632 (2006) \\
\hline UDP-polypeptide GalNAc-transferase & RT-PCR/bone marrow & Molecular marker of metastatic NB & Clin Chem; 52:1701-1712 (2006) \\
\hline \multicolumn{4}{|l|}{13 (GaINAc-T13 - GALNT13) } \\
\hline UDP-polypeptide GalNAc-transferase & RT-PCR/primary tumor & Prognostic marker (better outcome) & Clin Chem; 59(1):225-233 (2013) \\
\hline$\beta 1,4-N$-acetylgalactosaminyltransferase & $\mathrm{IHC} /$ primary tumor & Prognostic marker (better outcome) & Am J Pathol; 179:1394-1404 (2011) \\
\hline \multicolumn{4}{|l|}{3 (B4GALNT3) } \\
\hline$\beta-1,4$-galactosyltransferase III (B4GALT3) & $\mathrm{IHC} /$ primary tumor & Prognostic marker (poor outcome) & Clin Cancer Res; 19:1705-1716 (2013) \\
\hline
\end{tabular}

ICC, immunocytochemistry; RT-PCR, reverse transcriptase-polymerase chain reaction; ECL, electrochemiluminescence; PB, peripheral blood; IHC, immunohistochemistry. 
may play a crucial role in promoting malignant behavior of colon cancer (140). The same research team has recently published that $\beta$-1,4-galactosyltransferase III (B4GALT3) overexpression in colorectal cancer cells suppressed cell migration, invasion, and adhesion, while B4GALT3 knockdown enhanced malignant cell phenotypes promoting cell migration and invasion (141). Surprisingly, an opposite situation was found for both enzymes in NB. Firstly $\mathrm{Hsu}$ et al. communicated that B4GALNT3 expression positively correlates with the differentiation status of NB, predicting a favorable prognosis for patients and suppressing the malignant phenotype in cell lines experiments via decreasing $\beta 1$ integrin signaling (142). By contrast, $\beta$ 1,4-galactosyltransferase III (B4GALT3) expression in NB tumors correlated with advanced clinical stage, unfavorable histology, and lower survival rate (143). In conclusion, B4GALNT3 in NB seems a good prognostic marker, while B4GALT3 was suggested as poor outcome marker. Further work is necessary to elucidate subjacent molecular mechanisms for these enzymes.

Conflict of Interest Statement: The authors declare that the research was conducted in the absence of any commercial or financial relationships that could be construed as a potential conflict of interest.

Received: 30 June 2014; accepted: 08 July 2014; published online: 25 July 2014.

Citation: Berois N and Osinaga E (2014) Corrigendum: Glycobiology of neuroblastoma: impact on tumor behavior, prognosis, and therapeutic strategies. Front. Oncol. 4:193. doi: 10.3389/fonc.2014.00193

This article was submitted to Molecular and Cellular Oncology, a section of the journal Frontiers in Oncology. Copyright $(\odot 2014$ Berois and Osinaga. This is an openaccess article distributed under the terms of the Creative Commons Attribution License (CC BY). The use, distribution or reproduction in other forums is permitted, provided the original author(s) or licensor are credited and that the original publication in this journal is cited, in accordance with accepted academic practice. No use, distribution or reproduction is permitted which does not comply with these terms. 